\title{
Inverse Relationship between Release Probability and Readily Releasable Vesicles in Depressing and Facilitating Synapses
}

\author{
Andrew G. Millar, Haymo Bradacs, Milton P. Charlton, and Harold L. Atwood \\ Department of Physiology, University of Toronto, Toronto, Ontario, Canada M5S 1A8
}

\begin{abstract}
We tested the hypothesis that the probability of vesicular exocytosis at synapses is positively correlated with the pools of readily releasable synaptic vesicles, as shown for mammalian neurons grown in tissue culture. We compared synapses of two identified glutamatergic neurons: phasic (high-output, depressing) and tonic (low-output, facilitating) crustacean motor neurons, which differ 100- to 1000-fold in quantal content. Estimates of vesicles available for exocytosis were made from depletion during forced release and from electron microscopic observation of vesicles docked at synaptic membranes near
\end{abstract}

active zones. Both measurements showed a significantly larger pool of readily releasable vesicles in facilitating synapses, despite their much lower quantal output during stimulation. Thus, the probability for release of docked vesicles is very much lower at facilitating synapses, and the presence of more docked vesicles does not predict higher synaptic release probability in these paired excitatory neurons.

Key words: quantal content; glutamatergic; phasic; tonic; rapid depletion; synaptic differentiation; release probability; presynaptic; exocytosis; crustacean
The strength of synaptic transmission often differs among inputs to an innervated target in the CNS. Many synapses can be broadly classified as facilitating (having a low probability of initial transmitter release and exhibiting facilitation of release during trains of stimuli) or depressing (with high probability of initial release and depression of release during trains). Several examples have been described in the mammalian CNS, including parallel fiber (facilitating) and climbing fiber (depressing) inputs onto cerebellar Purkinje cells (Eccles et al., 1966; Konnerth et al., 1990; Dittman et al., 2000) and lateral (facilitating) and medial (depressing) perforant path synapses onto hippocampal granule cells (McNaughton, 1980). Understanding the mechanisms governing differences in presynaptic strength at facilitating and depressing synapses is important for our comprehension of neural networks and the brain as a whole.

Individual factors that contribute to the differences between facilitating and depressing synapses include those that determine the initial amount of transmitter released, which is generally higher for depressing than for facilitating synapses. The original model of del Castillo and Katz (1954) describing mean quantal content $(m)$ remains valid: $m=n p$, where $n$ represents mean number of units available for release at active synapses and $p$ represents the mean probability of release. The mean number of readily releasable synaptic vesicles and the mean probability of release of these vesicles could determine $n$ and $p$, respectively (del Castillo and Katz, 1954; Dittman et al., 2000).

Several recent studies have shown that for cultured mammalian

Received June 21, 2002; revised Aug. 27, 2002; accepted Aug. 29, 2002.

This research was supported by grants from the Canadian Institutes for Health Research (CIHR) to M.P.C. and H.L.A. and by a studentship from the CIHR to A.M. Some of the serial electron micrographs were photographed by Prof. C. K. Govind and Joanne Pearce (University of Toronto). Dr. Leo Marin provided advice and assistance for analysis of electron micrographs, and Ken Dawson-Scully provided a macro routine for measurement of vesicle distances. M. HegströmWojtowicz assisted with manuscript preparation.

Correspondence should be addressed to Andrew Millar, Department of Physiology, Medical Sciences Building, University of Toronto, Toronto, Ontario, Canada M5S 1A8. E-mail: andrew.millar@utoronto.ca.

Copyright (® 2002 Society for Neuroscience 0270-6474/02/229661-07\$15.00/0 neurons, the amount of transmitter initially released is directly proportional to the size of the readily releasable pool (RRP) of synaptic vesicles (Stevens and Tsujimoto, 1995; Rosenmund and Stevens, 1996; Dobrunz and Stevens, 1997; Schikorski and Stevens, 1999). To examine the generality of this conclusion, we tested the hypothesis that large differences in transmitter release probability at paired facilitating and depressing synapses innervating the same postsynaptic target are correlated with the size of the RRP.

Crustacean phasic and tonic motor neurons (Kennedy and Takeda, 1965a,b) provide an excellent system for the study of differences between facilitating and depressing synapses. With repeated stimulation, phasic synapses depress, whereas tonic synapses facilitate (Atwood and Wojtowicz, 1986). Furthermore, synaptic differentiation is extreme: initial release is 100 - to 1000 fold greater for phasic synapses than for tonic synapses (Msghina et al., 1998). Such extreme differences in release probability might be linked to greater numbers of readily releasable vesicles at phasic synapses; in turn, this feature could confer greater initial synaptic strength.

We tested this hypothesis with both physiological and ultrastructural methods. We estimated RRP sizes in tonic and phasic varicosities using a rapid depletion technique (Schneggenburger et al., 1999; Dittman et al., 2000). With electron microscopy, we determined the number of vesicles morphologically docked at the presynaptic active zone; these docked vesicles could correspond to the RRP (Schikorski and Stevens, 2001b). Contrary to our initial hypothesis, these measurements showed that tonic terminal varicosities possess more readily releasable vesicles than phasic varicosities and have twice as many docked vesicles per synapse. Estimated overall probability of vesicle release is 1500 -fold greater for phasic synapses. It is clear that vesicle release probability, and not the number of docked vesicles, determines initial synaptic strength in these glutamatergic neurons.

\section{MATERIALS AND METHODS}

Animals and preparation. Freshwater crayfish (Procambarus clarkii; 2-4 $\mathrm{cm}$ body length) were obtained from the Atchafalaya Biological Supply 
Company (Raceland, LA) and maintained under standard laboratory conditions (Bradacs et al., 1997). The extensor of the carpopodite (main leg extensor muscle), innervated by two excitatory motor axons, one phasic and one tonic, was selected for experimentation. The general anatomical and physiological features of the extensor muscle and its preparation have been described previously (Bradacs et al., 1997; Msghina and Atwood, 1997). The preparation was dissected and maintained in a modified Van Harreveld's crayfish solution containing (in $\mathrm{mm}$ ): $205.3 \mathrm{NaCl}, 5.3 \mathrm{KCl}, 13.5 \mathrm{CaCl}_{2} \cdot 2 \mathrm{H}_{2} \mathrm{O}, 2.5 \mathrm{MgCl}_{2} \cdot 6 \mathrm{H}_{2} \mathrm{O}$, and HEPES buffer at a pH of 7.4. To minimize contractions, the muscle was stretched after the membrane connecting the meropodite and carpopodite had been cut, leaving the tendon attachment intact. Experiments were performed at room temperature $\left(19-20^{\circ} \mathrm{C}\right)$.

Electrophysiological recording. We estimated the size of the RRP of quanta from well defined nerve terminal boutons and related this value to the amount of transmitter released by a single action potential. Neurotransmitter release was assayed by extracellular macropatch recording at visualized phasic and tonic nerve terminal boutons. The procedures were similar to those described for this preparation in Msghina et al. (1998). Focal macropatch recordings (Dudel, 1981; Wojtowicz et al., 1994; Cooper et al., 1995b) of the presynaptic nerve terminal action potential and excitatory junctional current (EJC) were obtained from single tonic and phasic terminal boutons made visible in preparations exposed briefly to the vital dye 4-(4-diethylaminostyryl)- $N$ methylpyridiniumiodide (4-Di-2-Asp; Molecular Probes, Eugene, OR) for $3 \mathrm{~min}$ at a concentration of $2 \mu \mathrm{M}$ (Cooper et al., 1995a,b).

The macropatch electrode, pulled from Kimax glass (outer diameter, $1.5 \mathrm{~mm}$ ), was beveled at $30^{\circ}$ with a Sutter electrode beveler (BV-10-B; Sutter Instruments, Novato, CA) until a tip diameter of $10-15 \mu \mathrm{m}$ was attained. The electrode tip was smoothed by fire-polishing to avoid damage to the neuromuscular junction. The electrode, filled with standard physiological solution, was placed under visual control over a well defined tonic or phasic bouton.

The excitatory axons were stimulated by pulses $(0.4 \mathrm{msec})$ delivered through a microelectrode $(20 \mathrm{M} \Omega$, filled with $3 \mathrm{M} \mathrm{KCl})$ inserted into either the phasic or tonic preterminal axon. Current was applied through an amplifier (model IE-201; Warner Instruments, Hamden, CT) operated in bridge mode, so that the presynaptic action potential could be observed when threshold was reached.

Data for transmitter release evoked by a single action potential were collected by stimulating either the phasic or tonic axon at $0.1 \mathrm{~Hz}(200$ trials) while recording EJCs from a visualized terminal bouton. An estimate of unitary quantal size was made by averaging 15-20 spontaneously occurring miniature EJCs (sEJCs) collected from the same bouton.

To estimate the number of readily releasable vesicles from the same bouton, we used a rapid depletion technique in which a train of stimuli produced synaptic depression. This method for estimating the RRP was based on the method used by Schneggenburger et al. (1999). A $200 \mathrm{~Hz}$ train (lasting 100 or $200 \mathrm{msec}$ ) of stimuli was applied to the presynaptic axon while EJCs were recorded from the selected nerve terminal bouton. Each stimulus caused a presynaptic action potential. Stimulus trains were repeated 30 times with a $60 \mathrm{sec}$ rest period between each trial. Because the facilitated synapses used here do not depress, the usual methods for estimating RRP could not be used. To ensure rapid depression, we increased transmitter release by broadening the presynaptic action potential. The $\mathrm{KCl}$ in the crayfish solution was replaced with $5.3 \mathrm{~mm} \mathrm{CsCl}$ (Atwood and Lang, 1973).

Data collection and analysis. Electrophysiological signals were low-pass filtered at $5 \mathrm{kHz}$ using an Intronix 2004-F Signal Conditioner (Intronix Technologies, Toronto, Canada) and subsequently digitized at $20 \mathrm{kHz}$ using a PowerLab/4sp (ADInstruments, Round Rock, TX) data acquisition system. Quantal content for each EJC was estimated by dividing the area of the evoked EJC by the area of the average SEJC (Cooper et al., 1995b) using the Scope data collection and analysis program (ADInstruments). Quantal content was estimated for unitary EJCs under control conditions and for each EJC in a train of depleting stimuli.

To estimate the number of readily releasable vesicles for a given bouton, we plotted the cumulative quantal content of EJCs during the $200 \mathrm{~Hz}$ train. A linear function was fitted to the last five events and extrapolated back to the $y$-axis. The $y$-intercept of this line was used as the estimate of the number of quanta in the RRP (Schneggenburger et al., 1999). The released fraction (the percentage of quanta released from the releasable pool by a single action potential) was calculated by dividing the unitary quantal content by the size of the RRP.

Ultrastructural measurements. Electron microscopy of phasic and tonic

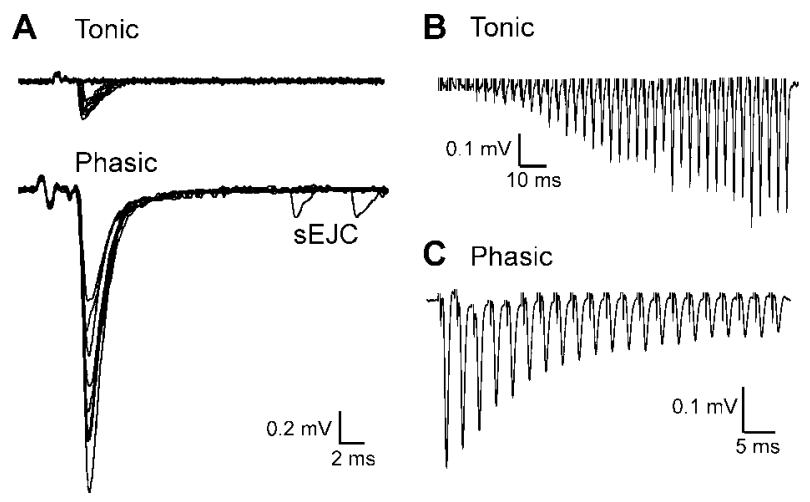

Figure 1. Comparison of synaptic release at low frequencies for phasic and tonic nerve terminals. $A$, EJCs recorded from tonic and phasic nerve terminal varicosities stimulated at $1 \mathrm{~Hz}$. Presynaptic stimulation evoked multiquantal EJCs from phasic terminals, whereas at tonic terminals, many stimuli evoked no release (failures), and some stimuli evoked an EJC similar in size to an sEJC. sEJCs are similar in size for both types of terminals, suggesting no large postsynaptic differences. $B, C$, Transmitter release in response to high-frequency trains of stimuli at tonic and phasic terminals. Traces shown are averages of 30 trials. Stimulus artifacts have been removed. $B$, Tonic terminal, showing marked facilitation of the EJC during a $200 \mathrm{~Hz}$ train of action potentials. No depression is evident. $C$, Phasic terminal, showing rapid depression of the EJC during a $200 \mathrm{~Hz}$ train of action potentials. EJCs reach a steady-state amplitude after $\sim 15$ stimuli.

boutons was used to estimate the number of synaptic vesicles at presynaptic membranes. The material was fixed and sectioned as described in previous publications on the crayfish extensor muscle (King et al., 1996; Bradacs et al., 1997; Msghina et al., 1998). Each synapse selected for study was sectioned serially (usually $\sim 10$ sections per synapse). Synapses selected for analysis met the following criteria: good resolution of synaptic vesicles and of presynaptic active zones possessing a dense body (Jahromi and Atwood, 1974; King et al., 1996) and absence of nearby (within $500 \mathrm{~nm}$ ) large intracellular organelles, especially mitochondria, which would displace synaptic vesicles. Quantitative analysis used the Sigma Scan program (SPSS, Chicago, IL). Synaptic vesicles were counted in a region bordered by the presynaptic membrane, by a line drawn parallel to the membrane at $500 \mathrm{~nm}$, and by lines drawn at right angles to the presynaptic membrane from its edges. The vesicles within this defined volume (summed for all sections of a serially sectioned synapse) were counted and used as an estimate of the total vesicle pool. The synaptic volumes in which vesicles were counted were similar for phasic and tonic synapses. For each synaptic vesicle, the closest linear distance to the presynaptic membrane was calculated by the program. Vesicles touching the presynaptic membrane or within $20 \mathrm{~nm}$ of it were included in the morphological estimate of docked vesicles. The value of $20 \mathrm{~nm}$ is derived from the likelihood of a vesicle reaching the presynaptic membrane within the minimal synaptic delay time of 200-500 $\mu \mathrm{sec}$ (Parsegian, 1977) and also represents the distance at which the membrane of a vesicle can just be resolved from the presynaptic membrane.

\section{RESULTS}

\section{Unitary quantal content}

Before the size of the RRP of vesicles for a defined phasic or tonic bouton was estimated, we determined the amount of neurotransmitter released from that particular bouton by a single presynaptic action potential. This estimation is called unitary quantal content. After nerve terminals were stained lightly with 4-Di-2Asp, a focal macropatch electrode (diameter, 10-15 $\mu \mathrm{m}$ ) was placed over either a tonic or a phasic nerve terminal bouton. EJCs were recorded from the selected bouton. We measured transmitter release at a stimulation rate of $0.1 \mathrm{~Hz}$ to avoid depression or facilitation. Examples from both tonic and phasic boutons are given in Figure $1 A$.

When stimulated at $0.1 \mathrm{~Hz}$, phasic boutons always responded 


\begin{tabular}{|c|c|c|c|}
\hline Experiment & $\begin{array}{l}\text { Unitary quantal } \\
\text { content }\end{array}$ & RRP size & $\begin{array}{l}\text { Released } \\
\text { fraction }(\%)\end{array}$ \\
\hline Phasic 1 & 15.6 & 74 & 21 \\
\hline Phasic 2 & 14.3 & 58 & 25 \\
\hline Phasic 3 & 17.8 & 42 & 42 \\
\hline Phasic 4 & 16.5 & 50 & 33 \\
\hline Phasic 5 & 17.1 & 66 & 26 \\
\hline Mean \pm SEM & $16.26 \pm 0.61$ & $58 \pm 5.6$ & $29 \pm 3.9$ \\
\hline Tonic 1 & 0.05 & 210 & 0.024 \\
\hline Tonic 2 & 0.015 & 80 & 0.019 \\
\hline Tonic 3 & 0.04 & 145 & 0.028 \\
\hline Tonic 4 & 0.025 & 122 & 0.020 \\
\hline Tonic 5 & 0.05 & 95 & 0.053 \\
\hline Mean \pm SEM & $0.036 \pm 0.007$ & $130.4 \pm 23$ & $0.02 \pm 0.006$ \\
\hline Phasic/tonic ratio & 451.67 & 0.44 & 1450.00 \\
\hline
\end{tabular}

with relatively large EJCs, which were several times larger than asynchronously released EJCs (Fig. 1A). After 200 trials at $0.1 \mathrm{~Hz}$ were performed, we recorded spontaneous transmitter release in the form of sEJCs, which are thought to result from transmitter released from a single vesicle quantal event. Unitary quantal content could then be estimated by dividing the mean area of the evoked EJC by the mean area of the sEJC (Cooper et al., 1995b). The quantal content of the evoked EJC for selected phasic terminal boutons ranged from 14 to 18 (Table 1).

In contrast, tonic terminal boutons released much less neurotransmitter when stimulated at $0.1 \mathrm{~Hz}$. For many stimuli, tonic boutons exhibited a nerve terminal action potential but did not produce an EJC. This indicated that the bouton was invaded by a presynaptic action potential, but transmitter release did not occur; we call this a failure of evoked release (Fig. $1 A$ ). The frequency of evoked EJCs ranged from 3 to 10 events over 200 stimuli. Because sEJCs were very similar in size to evoked EJCs, almost all evoked EJCs were composed of single quanta. Estimated unitary quantal content ranged from 0.015 to 0.05 for tonic boutons (Table 1). Overall, unitary quantal content was $\sim 450$ times greater for phasic boutons. These results are similar to those found previously by Msghina et al. (1998, 1999).

\section{Estimation of RRPs}

For both neurons, we estimated the size of the RRPs of vesicles in the selected bouton and correlated this with the unitary quantal content of the same bouton. Our estimate of the RRP was based on the rapid depression technique used for mammalian synapses by Schneggenburger et al. (1999). Generally, it is thought that during a rapid train of stimuli, the RRP is depleted and transmitter release is depressed. Any transmitter release that remains after the initial depression is a result of pool replenishment and continuing release, which come into equilibrium after depletion of the RRP (Elmquist and Quastel, 1965; Hubbard et al., 1969). If one factors out the transmitter release caused by pool replenishment, the remaining release must be from the RRP (Von Gersdorff et al., 1997; Dittman and Regehr, 1998; Wang and Kaczmarek, 1998; Schneggenburger et al., 1999).

The depletion method was applied readily to phasic boutons. In response to a $200 \mathrm{~Hz}$ burst of action potentials evoked by stimulation of the phasic axon, marked depression of transmitter re-
A

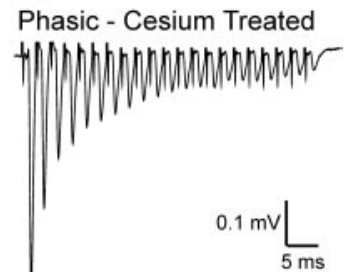

B

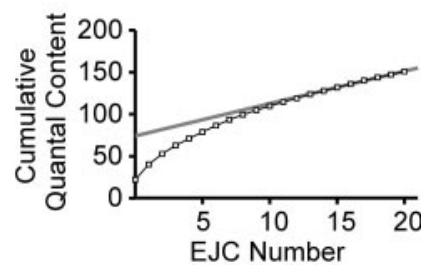

Figure 2. Estimation of RRP size at a phasic terminal. $A, \mathrm{~A} \mathrm{Cs}^{+}$containing solution was applied to the phasic terminal illustrated in Figure $1 \mathrm{~B}$, and $200 \mathrm{~Hz}$ trains $(100 \mathrm{msec})$ were applied (trace shown is the average of 30 trials). The $\mathrm{Cs}^{+}$treatment caused a slight increase in initial release, and EJCs reached a steady-state amplitude after $\sim 10$ stimuli. $B$, Cumulative plot of quantal content for the same phasic terminal during a $200 \mathrm{~Hz}$ train. Quantal content of each EJC of the train in $A$ was determined, and a linear regression line was fitted to the last five events, which represented the steady-state component of the train. Back-extrapolation to the start of the train gave an estimate of the number of quanta released before pool replenishment. In this example, 74 quanta were estimated for the RRP.

lease at the selected phasic boutons was always observed. In the example given in Figure $1 C$, the EJC amplitude became depressed to $\sim 20 \%$ of its initial value within 15 stimuli.

For normal tonic boutons, rapid depression could not be produced. When a $200 \mathrm{~Hz}$ burst of stimuli was applied to the tonic axon, facilitation of transmitter release was always observed at selected boutons (Fig. 1B). Because the depletion technique relies on rapid depression of transmitter release to estimate the RRP size, we had to modify the method to force tonic boutons to release transmitter at a rate high enough to obtain rapid depression. Atwood and Lang (1973) showed that replacing the $\mathrm{K}^{+}$in standard crustacean saline with $\mathrm{Cs}^{+}$produces a large increase in transmitter release from tonic terminals by causing the duration of the presynaptic action potential to increase. We therefore used $\mathrm{Cs}^{+}$treatment to increase the rate of depression in tonic terminals.

Application of $\mathrm{Cs}^{+}$to phasic nerve terminals did not have a large affect on the amount of transmitter released or the rate of depression. In the example given in Figure $2 A$, the same phasic terminal as in Figure $1 C$ was exposed to $\mathrm{Cs}^{+}$. The initial EJC was $\sim 30 \%$ larger than for the control; similar increases in initial EJC size were seen in the other phasic trials. The rate of depression in this case was slightly more rapid than before $\mathrm{Cs}^{+}$treatment, with EJCs depressing to $\sim 15 \%$ of initial value after 10 stimuli. Thereafter, EJC amplitudes remained relatively constant, indicating that a steady state of transmitter release had occurred.

When tonic terminals were incubated with $\mathrm{Cs}^{+}$saline, a large increase in neurotransmitter release occurred during $200 \mathrm{~Hz}$ bursts. The tonic bouton of Figure $1 B$ exhibited initial but transient facilitation of release after $\mathrm{Cs}^{+}$treatment, with EJCs reaching their maximum amplitude at the fourth stimulus (Fig. 3A). For all $\mathrm{Cs}^{+}$-treated tonic boutons, transient initial facilitation was followed by marked depression, in contrast to the progressive facilitation seen in controls (Fig. $1 B$ ). In Figure $3 A$, EJCs depressed to $\sim 20 \%$ of their maximum value after 30 stimuli. As was the case for phasic terminals, subsequent EJCs were relatively constant in amplitude, indicating steady-state release.

We have several lines of evidence to suggest that the depression we observed is not caused by postsynaptic glutamate receptor desensitization and/or saturation. First, according to Dudel et al. (1990), crayfish quisqualate-type glutamate receptors desensitize with a time constant of $6 \mathrm{msec}$ and recover totally from complete 
A

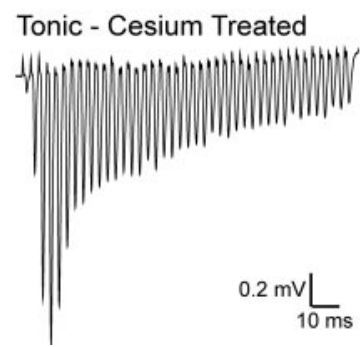

B

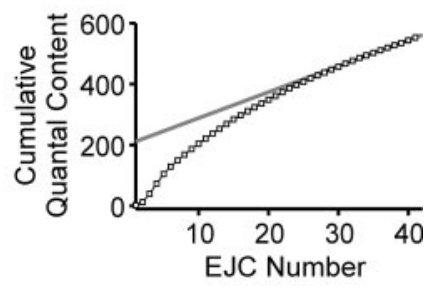

Figure 3. Estimation of RRP for a tonic terminal. $A, \mathrm{~A} \mathrm{Cs}^{+}$-containing solution was applied to the tonic terminal illustrated in Figure $1 C$, and 200 $\mathrm{Hz}$ trains $(200 \mathrm{msec}$ ) of stimuli were applied (trace shown is the average of 30 trials). The $\mathrm{Cs}^{+}$treatment greatly enhanced initial release, and maximal release was reached with four stimuli. Marked depression of release led to a steady-state amplitude after 30 stimuli. $B$, Cumulative plot of quantal content for the same tonic bouton during a $200 \mathrm{~Hz}$ train. Quantal content of each EJC of the train in $A$ was determined, and a cumulative plot was generated. A linear regression line was fitted to the last five events, which represented the steady-state component of the train. Back-extrapolation to the start of the train gave an estimate of the number of quanta released before pool replenishment. In this example, 210 quanta were estimated for the RRP.

desensitization within $3 \mathrm{msec}$. Because the EJCs we observe at these junctions are 2-3 msec in duration, the receptors will be only partially desensitized $(\sim 15 \%)$. Thus, with a stimulation interpulse period of $5 \mathrm{msec}(200 \mathrm{~Hz})$, receptors are allowed a 2-3 msec period for recovery from desensitization and therefore will be very close to a control state at the time of the next glutamate release. In short, recovery from receptor desensitization is so rapid that desensitization cannot be the cause of such a large depression. Second, to verify that there was not an additional slower process of recovery from desensitization or saturation, we measured sEJC integrals before and within $10 \mathrm{msec}$ of the end of our stimulus trains. A postsynaptic sensitivity change would be exhibited as a change in the SEJC integral, which is a measure of the postsynaptic response to a single quantum of neurotransmitter. There was no significant difference in sEJC integrals before and after stimulus trains in either phasic terminals $(-0.043 \pm$ 0.005 vs $-0.040 \pm 0.003 \mathrm{mV} \cdot \mathrm{mS} ; p>0.05 ; n=3)$ or tonic terminals $(-0.051 \pm 0.004 \mathrm{vs}-0.048 \pm 0.005 \mathrm{mV} \cdot \mathrm{mS} ; p>0.05$; $n=3$ ). Third, evidence put forth by Atwood et al. (1999) suggests that, at the similar Drosophila glutamatergic neuromuscular junction, fields of postsynaptic receptors are not saturated during normal quantal release. They show that variation of quantal amplitudes is too great for receptors to be saturated during normal release.

Because transmission could be depressed rapidly in $\mathrm{Cs}^{+}$treated boutons of both phasic and tonic neurons, we used the procedure of Schneggenburger et al. (1999) to estimate RRP sizes for both types of terminal boutons. For each EJC within a train, we calculated the quantal content; from these values, we generated a cumulative plot of released quanta (Figs. $2 B, 3 B$ ). To estimate the number of quanta in the RRP, we fitted a linear regression to the steady-state release portion of the cumulative plot and extrapolated this line back to the $y$-axis. The $y$-intercept gave an estimate of the number of quanta released in the absence of pool refilling or the number of quanta in the RRP. In Figure $2 B$, the estimated RRP for a phasic bouton was $\sim 74$ quanta. For a tonic bouton (Fig. $3 B$ ), the estimated RRP was $\sim 210$ quanta. These examples were representative: tonic boutons always had larger RRPs than phasic boutons.

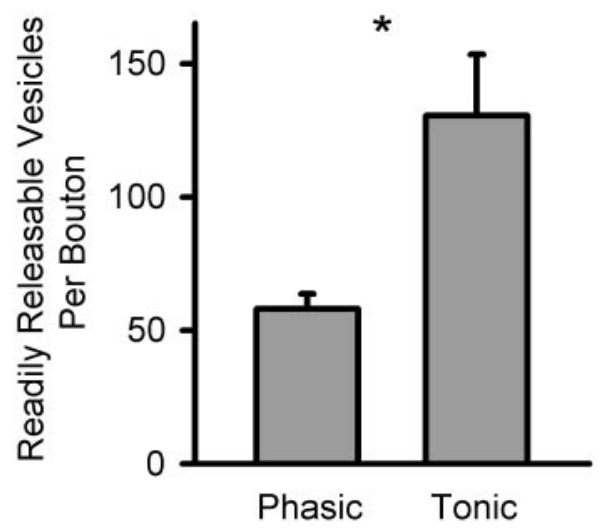

Figure 4. Mean RRP sizes for several tonic and phasic terminals. The mean RRP for tonic $(n=5)$ and phasic $(n=5)$ nerve terminal varicosities is given. Error bars indicate SEM. Asterisk, Significant difference $(p<$ 0.01 ; Student's $t$ test).

This analysis showed that tonic terminal boutons had more than twice as many quanta in their RRPs as phasic boutons (tonic, $130.4 \pm 23$; phasic, $58 \pm 5.6 ; p<0.01$ ) (Fig. 4, Table 1). From these results, we could determine the released fraction (the percentage of the RRP released by a single action potential) for each sampled bouton. This represents the relative probability of individual vesicle release (Schneggenburger et al., 1999). Table 1 shows that the released fractions for phasic and tonic boutons are vastly different; on average, a single action potential releases $30 \%$ of the RRP from phasic terminals and only $0.02 \%$ of the RRP from tonic terminals, a 1500-fold difference.

\section{Ultrastructural measurement of docked vesicles}

Morphological correlates of the RRP were sought through ultrastructural analysis. Individual phasic and tonic synapses were sectioned serially to determine the numbers of morphologically docked vesicles (Fig. $5 A$ ). Docked vesicles were defined as those touching the presynaptic membrane or within $20 \mathrm{~nm}$ of it (Fig. $5 B$ ).

Tonic synapses contained two to three times more morphologically docked vesicles than phasic synapses (tonic, $11.14 \pm 0.5$; phasic, $4.38 \pm 0.3 ; p<0.01$ ) (Fig. 5C, Table 2). When the number of docked vesicles was normalized to the contact area of the synapse, tonic synapses had twofold more docked vesicles.

Changing the distance criterion of a docked vesicle from 20 to $50 \mathrm{~nm}$ had no effect on the previous result; tonic synapses still contained two to three times more docked vesicles.

To compare morphologically docked vesicles and RRPs, we used docked vesicle counts of individual synapses to estimate docked vesicles for an entire bouton, this being the structure from which RRP measurements were made. The sizes of the terminal boutons selected for RRP determinations were measured with a micrometer. Using data from serial section studies by King et al. (1996) and Msghina et al. (1998), we estimated the number of synapses per bouton. Multiplying this value by the number of docked vesicles per synapse provided an estimate of docked vesicles per bouton. Tonic terminal boutons contained approximately seven times more docked vesicles than phasic boutons (Table 2). For phasic boutons, docked and readily releasable vesicle numbers were similar, with no significant difference. For tonic terminals, we estimated approximately three times more docked than readily releasable vesicles (Table 2 ). 
A

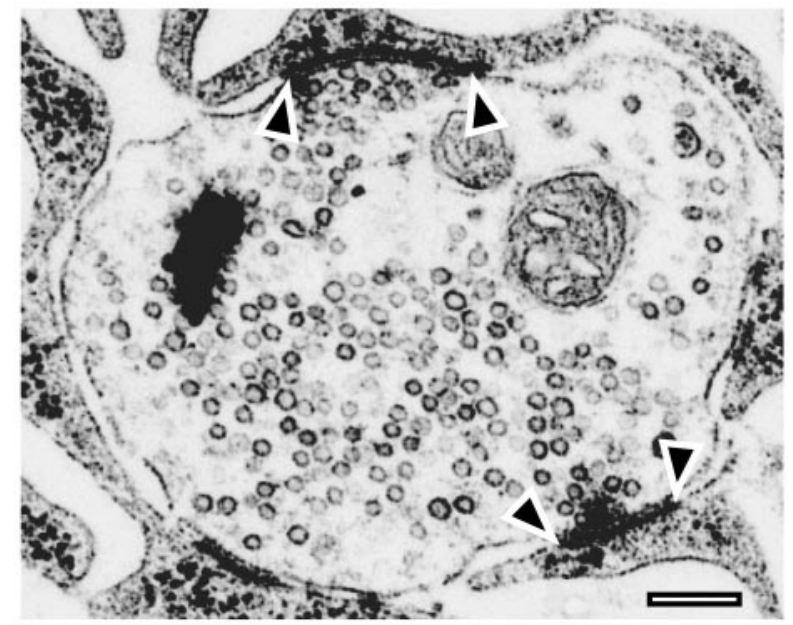

B
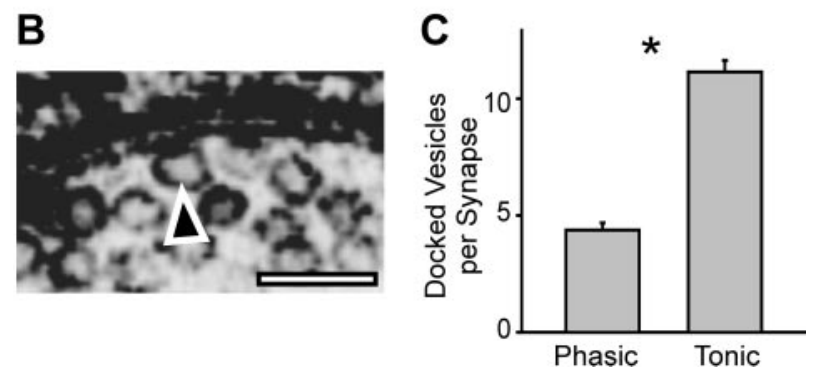

Figure 5. Estimates of docked vesicles from electron micrographs. $A$, Electron micrograph of a synapse-bearing tonic terminal, illustrating synapses (arrowheads) with vesicles clustered at a presynaptic dense bar of the active zone. Scale bar, $200 \mathrm{~nm}$. $B$, High-magnification micrograph of the upper synapse in $A$. The docked vesicle is marked with an arrowhead. Scale bar, $100 \mathrm{~nm}$. $C$, Comparison of estimates of docked vesicles at phasic and tonic synapses. The mean number of docked vesicles per synapse is given for tonic $(n=7)$ and phasic $(n=8)$ synapses. Error bars indicate SEM. Asterisk, Significant difference ( $p<0.01$; Student's $t$ test).

\section{DISCUSSION}

The hypothesis that the probability of vesicle exocytosis is determined by the size of the readily releasable vesicle pool was not supported by our results. Instead, the large differences in initial transmitter release at facilitating and depressing synapses appear to be governed by the probability of fusion of individual synaptic vesicles, which differs greatly in the two neurons. The facilitating tonic nerve terminals, with very low initial release, contain more than twice as many readily releasable and morphologically docked vesicles compared with the depressing phasic nerve terminals, which have high initial release. We estimate that a 1500 fold difference in vesicle release probability governs the initial release differences.

Previous work on crustacean tonic and phasic synapses supports this conclusion. Investigations of synaptic contact area have shown that morphological features of these synapses cannot explain release differences. In comparison with phasic terminals, tonic terminals contain more synapses per bouton, have generally larger individual synapses, and possess similarly sized active zones (King et al., 1996; Msghina et al., 1998, 1999). We have also ruled out major differences in calcium entry at active zones: the number of putative calcium channels at active zones and stimulus-
Table 2. Summary of morphological observations for phasic and tonic synapses

\begin{tabular}{llll} 
& Phasic & Tonic & $\begin{array}{l}\text { Phasic/tonic } \\
\text { ratio }\end{array}$ \\
\hline $\begin{array}{l}\text { Number of serially } \\
\text { sectioned synapses }\end{array}$ & 8 & 7 & \\
$\begin{array}{l}\text { Mean synaptic area } \\
\quad\left(\mu \mathrm{m}^{2}\right)\end{array}$ & $0.17 \pm 0.02$ & $0.19 \pm 0.02$ & \\
$\begin{array}{l}\text { Mean docked vesicles/ } \\
\text { synapse }\end{array}$ & $4.38 \pm 0.3$ & $11.14 \pm 0.5$ & \\
$\begin{array}{l}\text { Docked vesicles/ } \mu \mathrm{m}^{2} \\
\text { synapse area }\end{array}$ & $25.7 \pm 1.5$ & $58.6 \pm 2.6$ & 0.43 \\
$\begin{array}{l}\text { Synapses/ } \mu \mathrm{m} \text { of terminal } \\
\text { varicosity (from }\end{array}$ & & & \\
$\quad \begin{array}{l}\text { Msghina et al., } 1998) \\
\text { Mean length of terminal } \\
\text { varicosity (from light } \\
\text { microscopy data) }\end{array}$ & $1.23 \pm 0.28$ & $3.1 \pm 0.69$ & \\
$\begin{array}{l}\text { Estimated docked } \\
\text { vesicles/varicosity }\end{array}$ & $10.1 \pm 1.2$ & $10.4 \pm 2.3$ & \\
$\begin{array}{l}\text { Mean RRP sizes (from } \\
\text { Table 1) }\end{array}$ & 54 & 359 & 0.15 \\
\hline
\end{tabular}

evoked calcium entry per synapse were estimated to be similar for these synapses (Msghina et al., 1999). All results to date point to an intrinsic difference in the probability of release of individual vesicles.

In studies of the mammalian CNS, there has recently been much debate about whether the probability of initial release is set by the readily releasable vesicle pool size. Schikorski and Stevens (1997) demonstrated that for synapses of cultured hippocampal neurons, docked vesicle pool sizes and release probability are well correlated; however, direct measurements at identified synapses were not made. Dobrunz and Stevens (1997) demonstrated more directly a relationship of the RRP and initial release probability at single synapses of cultured hippocampal neurons. In contrast, Hanse and Gustafsson (2001), who measured physiological differences in hippocampal slice preparations, proposed that heterogeneity in synaptic release properties is correlated with vesicle release probability rather than with the size of the RRP. Also, a recent ultrastructural study (Xu-Friedman et al., 2001) showed that differences in synaptic response properties of cerebellar climbing and parallel fibers are not related to the docked vesicle pool size. These studies on synapses in situ are in agreement with our current findings. In addition, we directly related the readily releasable and docked vesicle pool sizes to initial release probability. Our study and others show that there is not always a direct correlation between RRP size and initial release probability for either mammalian or crustacean synapses. Thus, RRP size is not a universal mechanism accounting for synaptic quantal content. Presynaptic differentiation among synapses in situ apparently includes additional mechanisms.

The finding that factors governing vesicular release probability may be different in cultured and in situ neurons suggests that developmental and environmental influences determine synaptic response properties. Cultured neurons grow in a simplified environment in which normal trophic influences, activity-related modulation, and circulating messengers are absent. Development in a simplified environment may enhance the dependence of 
synaptic release on the RRP of synaptic vesicles. In contrast, normal development in situ may call forth additional synaptic regulatory mechanisms, leading to differences in synaptic molecular endowments.

In the second part of this study, we used morphologically docked vesicles as a possible correlate of the RRP. This assumption is supported in recent studies by Schikorski and Stevens (2001a,b), who established that docked and readily releasable vesicles are drawn from the same pool. In our study, we show that these two pools correlate well for phasic terminals. However, in tonic terminals, there were more docked vesicles than readily releasable vesicles. This difference could arise in several ways. First, it is generally thought that vesicles must be both docked and "primed" to undergo immediate exocytosis (for review, see Klenchin and Martin, 2000). Tonic synapses probably contain a large proportion of docked but unprimed vesicles. The need for additional priming at tonic synapses is demonstrated in Figure 3, which shows a delay in attaining maximal release. Second, in tonic terminals, a number of the synapses on a bouton are "silent;" that is, they contain vesicles but do not provide the conditions for normal impulse-evoked exocytosis (for review, see Atwood and Wojtowicz, 1999; Quigley et al., 1999). Such silent synapses contain vesicles that would be counted as docked but would not contribute to the RRP of vesicles for that bouton.

Although we have now ruled out the hypothesis that differences in RRP size govern release differences at phasic and tonic synapses, the question as to what factors govern the extreme physiological differentiation remains. All available evidence suggests that differentiation must occur at the level of individual vesicle exocytosis. Vesicles at tonic synapses are apparently restrained more rigidly and require more priming before they can be released. Thus, there is most likely a difference in the sensitivity of the transmitter release mechanism to calcium: the phasic release machinery is probably more responsive to presynaptic calcium transients. Previous reports indicate that sensitivity of release to available calcium varies widely among different neurons (Heidelberger et al., 1994; Bollmann et al., 2000; Schneggenburger and Neher, 2000). If this were the case for phasic/tonic synaptic differentiation, a higher probability of individual vesicle release at phasic synapses could result from molecular differences in the calcium receptor mechanism governing vesicular fusion.

Finally, because phasic terminals modify their physiological and morphological properties to acquire a more tonic phenotype in response to conditioning stimulation (Lnenicka and Atwood, 1985; Lnenicka et al., 1986; Lnenicka and Hong, 1997), presynaptic changes related to neural circuit modification and learning mechanisms may include changes in probability of vesicular release.

\section{REFERENCES}

Atwood HL, Lang F (1973) Differential responses of crab neuromuscular synapses to cesium ion. J Gen Physiol 61:747-766.

Atwood HL, Wojtowicz JM (1986) Short-term and long-term plasticity and physiological differentiation of crustacean motor synapses. Int Rev Neurobiol 28:275-362.

Atwood HL, Wojtowicz JM (1999) Silent synapses in neural plasticity: current evidence. Learn Mem 6:542-571.

Atwood HL, Karunanithi S, Wong K, Marin L, Stewart BA (1999) Determinants of quantal size at Drosophila synapses: bouton type, vesicle size, and the size of the postsynaptic receptor patch. Soc Neurosci Abstr 25:475.

Bollmann JH, Sakmann B, Borst JGG (2000) Calcium sensitivity of glutamate release in a calyx-type terminal. Science 289:953-957.

Bradacs H, Cooper RL, Msghina M, Atwood HL (1997) Differential physiology and morphology of phasic and tonic motor axons in a crayfish limb extensor muscle. J Exp Biol 200:677-691.
Cooper RL, Marin L, Atwood HL (1995a) Synaptic differentiation of a single motor neuron: conjoint definition of transmitter release, presynaptic calcium signals, and ultrastructure. J Neurosci 15:4209-4222.

Cooper RL, Stewart BA, Wojtowicz JM, Wang S, Atwood HL (1995b) Quantal measurement and analysis methods compared for crayfish and Drosophila neuromuscular junctions, and rat hippocampus. J Neurosci Methods 61:67-78.

del Castillo J, Katz B (1954) Statistical factors involved in neuromuscular facilitation and depression. J Physiol (Lond) 124:574-585.

Dittman JS, Regehr WG (1998) Calcium dependence and recovery kinetics of presynaptic depression at the climbing fiber to Purkinje cell synapse. J Neurosci 18:6147-6162.

Dittman JS, Kreitzer AC, Regehr WG (2000) Interplay between facilitation, depression, and residual calcium at three presynaptic terminals. J Neurosci 20:1374-1385.

Dobrunz LE, Stevens CF (1997) Heterogeneity of release probability, facilitation, and depletion at central synapses. Neuron 18:995-1008.

Dudel J (1981) The effect of reduced calcium on quantal unit current and release at the crayfish neuromuscular junction. Pflügers Arch 391:35-40.

Dudel J, Franke CH, Hatt H (1990) Rapid activation, desensitization, and resensitization of synaptic channels of crayfish muscle after glutamate pulses. Biophys J 57:533-545.

Eccles JC, Llinás R, Sasaki K (1966) The excitatory synaptic action of climbing fibres on the Purkinje cells of the cerebellum. J Physiol (Lond) 182:268-296.

Elmquist D, Quastel DMJ (1965) A quantitative study of end-plate potentials in isolated human muscle. J Physiol (Lond) 178:505-529.

Hanse E, Gustafsson B (2001) Factors explaining heterogeneity in shortterm synaptic dynamics of hippocampal glutamatergic synapses in the neonatal rat. J Physiol (Lond) 5371:141-149.

Heidelberger R, Heinemann C, Neher E, Matthews G (1994) Calcium dependence of the rate of exocytosis in a synaptic terminal. Nature 371:513-515.

Hubbard JI, Llinas R, Quastel DMJ (1969) Electrophysiological analysis of synaptic transmission. London: Edward Arnold.

Jahromi SS, Atwood HL (1974) Three-dimensional ultrastructure of the crayfish neuromuscular apparatus. J Cell Biol 63:599-613.

Kennedy D, Takeda K (1965a) Reflex control of abdominal flexor muscles in the crayfish. I. The twitch system. J Exp Biol 43:211-227.

Kennedy D, Takeda K (1965b) Reflex control of abdominal flexor muscles in crayfish. II. The tonic system. J Exp Biol 43:229-246.

King MJR, Atwood HL, Govind CK (1996) Structural features of crayfish phasic and tonic neuromuscular junctions. J Comp Neurol 372:618-626.

Klenchin VA, Martin TF (2000) Priming in exocytosis: attaining fusioncompetence after vesicle docking. Biochimie 82:399-407.

Konnerth A, Llano I, Armstrong CM (1990) Synaptic currents in cerebellar Purkinje cells. Proc Natl Acad Sci USA 87:2662-2665.

Lnenicka GA, Atwood HL (1985) Age-dependent long-term adaptation of crayfish phasic motor axon synapses to altered activity. J Neurosci 5:459-467.

Lnenicka GA, Hong SJ (1997) Activity-dependent changes in voltagedependent calcium currents and transmitter release. Mol Neurobiol 14:1-31.

Lnenicka GA, Atwood HL, Marin L (1986) Morphological transformation of synaptic terminals of a phasic motoneuron by long-term tonic stimulation. J Neurosci 6:2252-2258.

McNaughton BL (1980) Evidence for two physiologically distinct perforant pathways to the fascia dentata. Brain Res 199:1-19.

Msghina M, Atwood HL (1997) Distribution and morphology of inhibitory innervation in crayfish limb and abdominal muscles. Cell Tissue Res 290:111-118.

Msghina M, Govind CK, Atwood HL (1998) Synaptic structure and transmitter release in crustacean phasic and tonic motor neurons. J Neurosci 18:1374-1382.

Msghina M, Millar AG, Charlton MP, Govind CK, Atwood HL (1999) Calcium entry related to active zones and differences in transmitter release at phasic and tonic synapses. J Neurosci 19:8419-8434.

Parsegian VA (1977) Considerations in determining the mode of influence of calcium on vesicle-membrane interaction. In: Society for Neuroscience symposia, Vol II, Approaches to the cell biology of neurons (Cowan WW, Ferrendelli JA, eds), pp 161-171. Bethesda, MD: Society for Neuroscience.

Quigley PA, Msghina M, Govind CK, Atwood HL (1999) Visible evidence for differences in synaptic effectiveness with activity-dependent vesicular uptake and release of FM1-43. J Neurophysiol 81:356-370.

Rosenmund C, Stevens CF (1996) Definition of the readily releasable pool of vesicles at hippocampal synapses. Neuron 16:1197-1207.

Schikorski T, Stevens CF (1997) Quantitative ultrastructural analysis of hippocampal excitatory synapses. J Neurosci 17:5858-5867.

Schikorski T, Stevens CF (1999) Quantitative fine-structural analysis of olfactory cortical synapses. Proc Natl Acad Sci USA 96:4107-4112.

Schikorski T, Stevens CF (2001a) Morphological correlates of functionally defined synaptic vesicle pools. Soc Neurosci Abstr 27:1009. 
Schikorski T, Stevens CF (2001b) Morphological correlates of functionally defined synaptic vesicle populations. Nat Neurosci 4:391-395.

Schneggenburger R, Neher E (2000) Intracellular calcium dependence of transmitter release rates at a fast central synapse. Nature 406:889-983.

Schneggenburger R, Meyer AC, Neher E (1999) Released fraction and total size of a pool of immediately available transmitter quanta at a calyx synapse. Neuron 23:399-409.

Stevens CF, Tsujimoto T (1995) Estimates for the pool size of releasable quanta at a single central synapse and for the time required to refill the pool. Proc Natl Acad Sci USA 92:846-849.
Von Gersdorff H, Schneggenburger R, Weis S, Neher E (1997) Presynaptic depression at a calyx synapse: the small contribution of metabotropic glutamate receptors. J Neurosci 17:8137-8146.

Wang L-Y, Kaczmarek LK (1998) High-frequency firing helps replenish the readily releasable pool of synaptic vesicles. Nature 394:384-388.

Wojtowicz JM, Marin L, Atwood HL (1994) Activity-induced changes in synaptic release sites at the crayfish neuromuscular junction. J Neurosci 14:3688-3702.

Xu-Friedman MA, Harris KM, Regehr WG (2001) Three-dimensional comparison of ultrastructural characteristics at depressing and facilitating synapses onto cerebellar Purkinje cells. J Neurosci 21:6666-6672. 